\title{
Femtosecond soliton spatio-temporal properties in multimode GRIN fibers
}

\author{
Mario Zitelli,(1) Fabio Mangini, (2) Mario Ferraro,(1) and Stefan Wabnitz ${ }^{(1)}$ \\ ${ }_{1}^{1}$ Department of Information Engineering, Electronics and Telecommunications (DIET), Sapienza \\ University of Rome, Via Eudossiana 18, 00184 Rome, Italy, mario.zitelli@uniroma1.it \\ 2 Department of Information Engineering, University of Brescia, Via Branze 38, 25123 Brescia, Italy
}

\begin{abstract}
Femtosecond multimode solitons composed by non-degenerate modes have unique properties: their pulsewidth and energy only depend on linear dispersive properties, and coupling conditions. They are unstable objects which evolve into stable singlemode pulses.
\end{abstract}

\section{Introduction}

Multimode fibers (MMFs) have recently attracted considerable interest, motivated by their potential for increasing the transmission capacity of long-distance optical links via modedivision-multiplexing (MDM), which exploits the multiple transverse modes of the fiber as information carriers. Moreover, the capacity of MMF to carry high-energy beams is important for scaling the power delivery capabilities of fiber laser sources. Graded-index (GRIN) MMFs are capable of of supporting the propagation of MM solitons over long distances ${ }^{[1]-[3]}$. Theoretical treatments of spatiotemporal soliton propagation in MMFs mostly rely on the variational approach $(V A)^{[4],[5]}$. When neglecting the temporal dimension, the variational method permits to include the Kerr effect in the description of periodic spatial self-imaging $(\mathrm{SSI})^{[6]-[9]}$. The validity of the VA is based on the assumption that the initial beam shape is maintained unchanged upon propagation, except for a limited set of slowly-varying parameters (e.g., the beam amplitude and width).

In this work, we reveal that the the generation of femtosecond MM soliton beams in GRIN fibers exhibits previously unexpected, and complex dynamics, which make them very different from their well-known singlemode counterparts. In singlemode fibers, a soliton forms when chromatic dispersion pulse broadening is compensated for by self-phase modulation. This means that a singlemode soliton can have an arbitrary temporal duration, provided that its peak power is properly adjusted, In contrast, the soliton condition in a MMF also requires the compensation of modal dispersion. This leads to the (previously un-noticed) property that MM solitons composed of non-degenerate modes have a fixed pulsewidth and energy at each wavelength, independently of the input pulse duration.

Moreover, we observed that, for long propagation distances, the beam content of MM solitons is irreversibly attracted toward the fundamental mode of the MMF. This is due to the combined action of intermodal four-wave mixing (FWM) and stimulated Raman scattering $(\mathrm{SRS})^{[10]}$. These results are of particular importance for different applications of nonlinear MMFs, such as high-power mode-locked laser sources, and soliton-based transmission.

\section{MM soliton modal attraction}

In order to guide experiments, we numerically simulated the propagation of MM pulses by means of a coupled-mode equation model[11],[12]. The fields of propagating modes are coupled by inter-modal FWM and SRS via mixing products of the type $Q_{p l m n} A_{1} A_{m} A_{n}{ }^{*}$, where $Q_{p l m n}$ are coupling coefficients proportional to the overlap integrals of transverse modal field distributions. The lack of symmetry between coupling coefficients and Raman gain are responsible for a slow, but irreversible transfer of energy from higher-order modes towards the fundamental mode. This occurs when pulses carried by different modes are both temporally and spatially overlapping.

Fig. 1 (left) shows the simulated energy evolution of the propagating $(1,0),(2,0)$, and $(3,0)$ modes with axial symmetry, when coupling at the fiber input a Gaussian pulse with $67 \mathrm{fs}$ pulsewidth, $1550 \mathrm{~nm}$ wavelength, $30 \mu \mathrm{m}$ diameter, and optimal peak power of $28 \mathrm{~kW}$. The top-right panel of Fig. 1 shows the input temporal profile of the power in the three modes; the bottom-right panel shows the corresponding output mode power.

Simulations (not shown here) show that nonlinear coupling among the three nondegenerate modes initially leads to the generation of a MM soliton pulse. Specifically, because of cross-phase modulation, the modes remain temporally trapped with each other, and propagate at the same speed, in spite of their 
linear group-velocity walk-off.

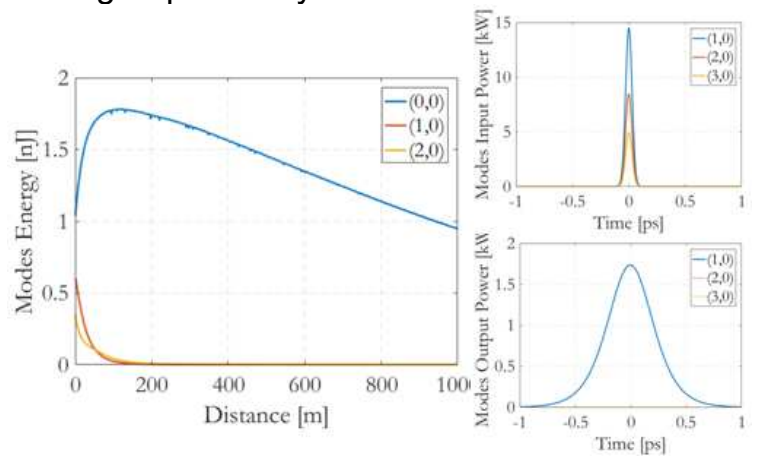

Fig. 1: Numerical simulation of 3 axial modes propagating over $1 \mathrm{~km}$ of GRIN fiber.

However, Fig. 1 shows that, after approximately $120 \mathrm{~m}$ of propagation, the fundamental mode acts as an attractor, and it acquires the energy carried by higher-order modes. Fig. 1 shows that, the subsequent $880 \mathrm{~m}$ of propagation, a substantially monomodal soliton propagates: this pulse experiences a continuous wavelength shift, owing to Raman soliton self-frequency shift (SSFS). As the soliton wavelength increases above $1700 \mathrm{~nm}$, it starts losing its power because of linear attenuation. This leads to its adiabatic temporal broadening, in order to preserve the soliton condition (bottom-right inset of Fig. 1).

To experimentally investigate multimode soliton generation and dynamics, we propagated femtosecond pulses for up to $850 \mathrm{~m}$ of multimode GRIN MMF. Pulses with 67 fs temporal duration at $1550 \mathrm{~nm}$, with Gaussian beam shape $\left(\mathrm{M}^{2}=1.3\right)$ and $1 / \mathrm{e}^{2}$ input diameter of approximately $30 \mu \mathrm{m}$, where injected at the fiber input. The MMF was a span of commercial GRIN fiber, with core radius $r_{c}=25 \mu \mathrm{m}$, cladding radius $r_{\text {clad }}=62.5 \mu \mathrm{m}$, cladding index $n_{\text {clad }}=1.444$, and relative index difference $\Delta=0.0103$.

Fig. 2 shows the dependence on input pulse energy of the measured output soliton pulsewidth, after $850 \mathrm{~m}$ of GRIN fiber. The different solid curves refer to the generation of different Raman solitons. As can be seen from the blue solid curve (first Raman soliton), there is an optimal input energy of 1.75-2.25 $\mathrm{nJ}$, which leads to the minimum pulsewidth at the MMF output (i.e., $450 \mathrm{fs}$ after $850 \mathrm{~m}$ ). For larger input energies, a fission of the input pulse into multiple solitons is observed, leading to the generation of a second and third Raman soliton. Fig. 3 illustrates the corresponding measured output beam waist, which reduces to a value that is close the fundamental mode waist $(8 \mu \mathrm{m})$ at the input energy for minimum pulsewidth. This confirms that a soliton which is only carried by the fundamental fiber mode has survived at the
GRIN MMF output.

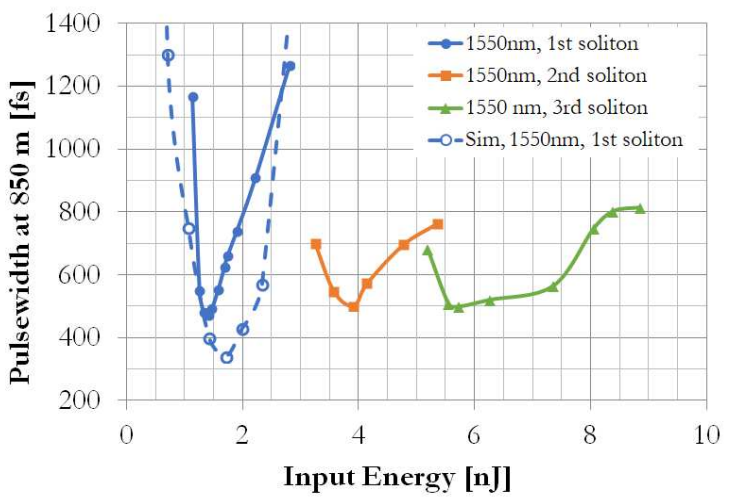

Fig. 2: Measured soliton pulsewidth at $1550 \mathrm{~nm}$, after $850 \mathrm{~m}$ of GRIN fiber.

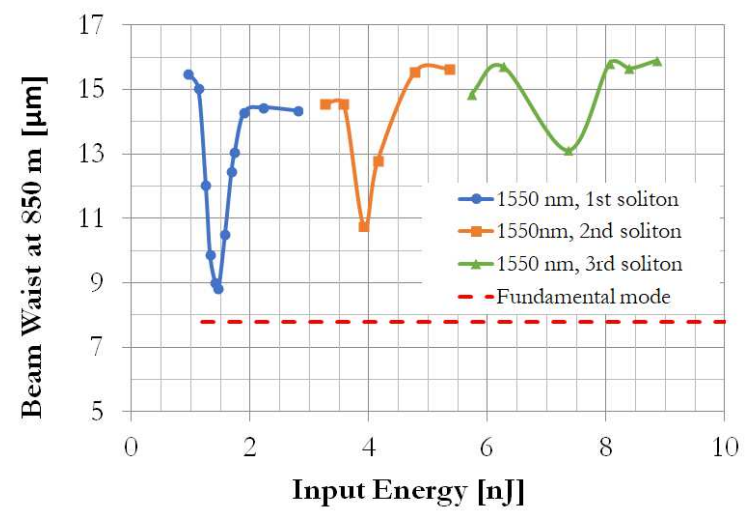

Fig. 3: Measured output beam waist after $850 \mathrm{~m}$ of GRIN fiber.

\section{MM soliton pulsewidth dynamics}

In order to analyze the details of the femtosecond multimode soliton generation process, we carried out extensive simulations and experiments for a series of fiber lengths (i.e., 1, 2, 6, 10, 20, 120, and $850 \mathrm{~m}$ ), and different input pulse wavelengths (i.e., 1300, $1350,1420,1550$, and $1680 \mathrm{~nm}$ ). We also varied the input pulsewidth from 60 up to $240 \mathrm{fs}$. In all cases, the input beam excitation consisted of the first three non-degenerate modes (i.e., $(1,0),(2,0)$, and $(3,0))$ with axial symmetry.

Fig. 4 shows the results of numerical simulations, illustrating the evolution with distance of the generated MM soliton pulsewidth. Here we considered several input wavelengths $(1350,1550$, and $1680 \mathrm{~nm})$ and two values of the input pulse widths (70 and 240 fs). On the other hand, for each pair of wavelength and pulse duration values, we selected the optimal input pulse energy which provides a stable long-range first Raman soliton (i.e., over $120 \mathrm{~m}$ and more), i.e., corresponding to a minimum pulse width as in Fig.2. Simulations show that, after just a few meters of propagation, the generated MM soliton has a fixed pulsewidth, independently of the input 
pulse duration.

We observed that the pulse width of the generated MM soliton is larger for increasing values of the input pulse wavelength. It was also found that the input optimal energy remains between 2 and $3 \mathrm{~nJ}$ for all input pulsewidths, with only a weak dependence on the input wavelength and coupling conditions.

Experiments confirmed well numerical predictions. We measured the soliton that is formed after long spans of GRIN fiber (i.e., either $120 \mathrm{~m}$ or $850 \mathrm{~m}$ ), when injecting pulses with optimal energy values between 2 and $3 \mathrm{~nJ}$. When repeating our experiments with shorter fibers at the same optimal energy, and input pulsewidths between 60 and 100 fs, we could measure the pulsewidth of the soliton at the distance where it is initially generated. For example, this occurs at $2 \mathrm{~m}$ for an input 1300 $\mathrm{nm}$ pulse, or at $6 \mathrm{~m}$ for other input wavelengths. Results are provided in Fig. 5 (green circles), together with corresponding numerical simulations for same input energy, and input pulsewidths of either 70 (blue saltires) or $240 \mathrm{fs}$ (red crosses).

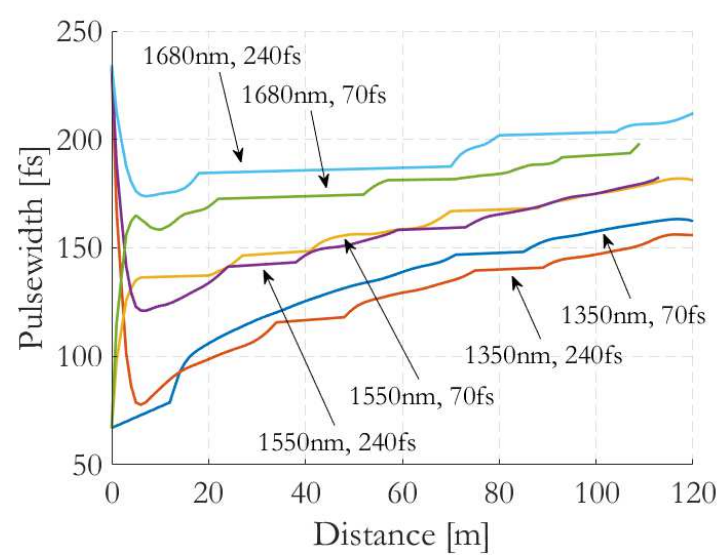

Fig. 4: Simulated MM soliton pulsewidth for 70 fs or $240 \mathrm{fs}$ duration, and 1350, 1550, or $1680 \mathrm{~nm}$ wavelength input pulses.

Fig. 5 reveals the peculiar properties of femtosecond MM solitons, which are composed by non-degenerate modes. As can be seen, the soliton pulsewidth only depends on its wavelength, and it remains nearly independent of the temporal duration of the input pulse. Whereas all MM solitons are generated for similar values of the input pulse energy.

In MMFs, soliton formation requires not only that chromatic dispersion and self-phase-modulation induced chirp balance each other, but also that cross-phase modulation compensates for modal dispersion. As a result, it is necessary that both dispersion and nonlinearity lengths are nearly equal to the fiber walk-off length ${ }^{[13]}: L_{D}=L_{N L}=$ const $\cdot L_{W}$, being const an adjustment constant; from this condition, it is possible to calculate the theoretical black curve of Fig. 5, which only depends only on the fiber dispersive parameters, hence the wavelength.

For femtosecond MM solitons, the nonlinear length is shorter than the random mode coupling and birefringence lengths, i.e., $L_{N L}<L_{c m}, L_{c p}$. In these conditions, the MM soliton irreversibly evolves into a fundamental-mode soliton at long distances.

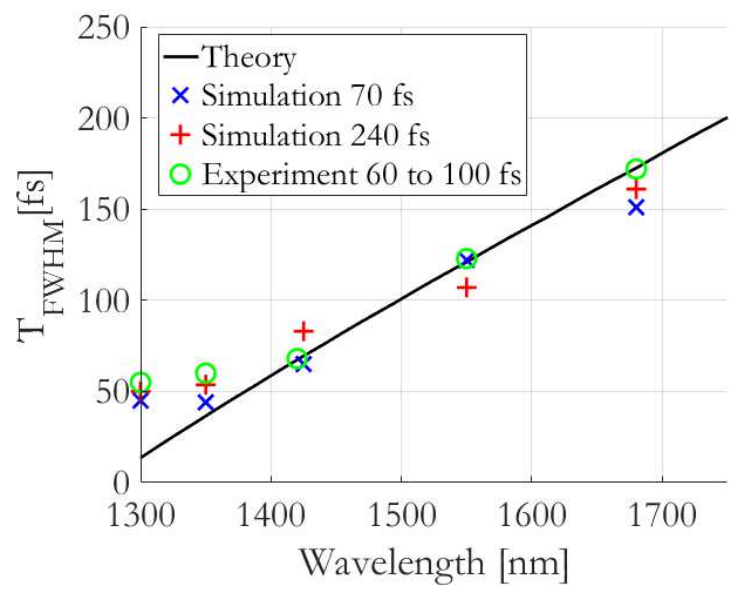

Fig. 5: Pulsewidth of forming MM soliton vs. wavelength, at the optimal input energy.

\section{Conclusions}

We have revealed that femtosecond $\mathrm{MM}$ solitons composed by non-degenerate modes have unique properties, which are quite different from their single-mode cousins. Multimode solitons form with an energy and pulsewidth which are nearly independent of the input pulse duration. Their generation mechanism is governed by a balance between modal walk-off, chromatic dispersion, and nonlinearity. Therefore, MM soliton temporal duration only depends on fiber dispersion and input wavelength. Moreover, it is not possible to generate non-degenerate mode MM solitons with durations longer than a few hundreds of fs. In addition, intermodal FWM and SRS lead to a progressive transfer of energy from higher-order modes into the fundamental mode.

\section{Acknowledgements}

We acknowledge the financial support from the European Research Council Advanced Grants No. 874596 and No. 740355 (STEMS), and the Italian Ministry of University and Research (R18SPB8227). 


\section{References}

[1] B. Crosignani and P. D. Porto, "Soliton propagation in multimode optical fibers," Opt. Lett., vol. 6, pp. 329-330, 1981.

[2] A. S. Ahsan and G. P. Agrawal, "Gradedindex solitons in multimode fibers," Opt. Lett., vol. 43, pp. 3345-3348, 2018.

[3] Renninger, W. H., \& Wise, F. W., Optical solitons in graded-index multimode fibres, Nat. Commun., vol. 4, pp. 1719, 2013.

[4] S.-S. Yu, C.-H. Chien, Y. Lai, and J. Wang, "Spatio-temporal solitary pulses in gradedindex materials with Kerr nonlinearity," Opt. Commun., vol. 119, pp. 167-170, 1995.

[5] S. Raghavan and G. P. Agrawal, "Spatiotemporal solitons in inhomogeneous nonlinear media," Opt. Commun., vol. 180, pp. 377 - 382, 2000.

[6] M. Karlsson, D. Anderson, and M. Desaix, "Dynamics of self-focusing and self-phase modulation in a parabolic index optical fiber," Opt. Lett., vol. 17, pp. 22-24, 1992.

[7] T. Hansson, A. Tonello, T. Mansuryan, F. Mangini, M. Zitelli, M. Ferraro, A. Niang, R. Crescenzi, S. Wabnitz, and V. Couderc, "Nonlinear beam self-imaging and selffocusing dynamics in a grin multimode optical fiber: theory and experiments," Opt. Express, vol. 28, pp. 24005-24021, 2020.

[8] F. Mangini, M. Ferraro, M. Zitelli, A. Niang, A. Tonello, V. Couderc, \& S. Wabnitz, "Multiphoton-Absorption-Excited UpConversion Luminescence in Optical Fibers," Physical Review Applied, vol. 14(5), 054063, 2020.

[9] M. Conforti, C. M. Arabi, A. Mussot, and A. Kudlinski, "Fast and accurate modeling of nonlinear pulse propagation in gradedindex multimode fibers," Opt. Lett., vol. 42, pp. 4004-4007, 2017.

[10] M. Zitelli, M. Ferraro, F. Mangini, S. Wabnitz, " Single-mode spatiotemporal soliton attractor in multimode GRIN fibers," Phot. Research, vol.9, no. 5, pp. 741-748, 2021.

[11] F. Poletti and P. Horak, "Description of ultrashort pulse propagation in multimode optical fibers," J. Opt. Soc. Am. B vol. 25(10), pp. 1645-1654, 2008.

[12] L. G. Wright, Z. M. Ziegler, P. M. Lushnikov, Z. Zhu, M. A. Eftekhar, D. N. Christodoulides, and F.W. Wise, "Multimode nonlinear fiber optics: Massively parallel numerical solver, tutorial, and outlook," IEEE J. Sel. Top. Quantum Electron., vol. 24, pp. 1-16, 2018.

[13] A. Hasegawa, "Self-confinement of multimode optical pulse in a glass fiber", 